\section{Die Aufgabe der Forschung in der medizinischen Versorgung von tragender Stute und neugeborenem Fohlen}

\author{
P. Rossdale \\ Beaufort Cottage Stables, Newmarket
}

\section{Einleitung}

Diese Veröffentlichung sei in ehrenvollem Gedenken Jobn und Sandy Hartigan gewidmet, die als Mitglieder des University of Florida's College of Veterinary Medicine and Equine Research Center durch ihre Forschungsarbeit wesentlich dazu beitrugen, die Haltungsbedingungen des Pferdes zu verbessern und die Erfolgsquote von Zuchtprogrammen zu erhöhen. Beide zeigten stets größten persönlichen Einsatz und praktisches Engagement in ihrem Bemühen, im Dienst der wissenschaftlichen Forschung tätig zu sein. Ihre Leistungsbereitschaft, Wissen, Begeisterung und Streben nach neuen Erkenntnissen waren Eigenschaften, die in der Forschung tätige Wissenschaftler als grundlegende Voraussetzung schon immer besitzen mußten. Auch heute und in Zukunft wird man ohne diese Attribute nicht auskommen, wenn man bei seiner Arbeit an die Grenzen des heutigen Wissensstandes stößt.

Die letzte Phase der Trächtigkeit und die erste Zeit nach der Geburt sind von entscheidender Bedeutung für die Entwicklung des Rennpferdes. Fötus und neugeborenes Fohlen sind die Vorläufer des Athleten, zu dem sich das Fohlen zwei Jahre später entwickelt haben soll (Abb. 1). Angehörige der veterinärmedizinischen und anderer wissenschaftlicher Fakultäten der Universität von Florida haben bedeutsame Beiträge zur Erweiterung der Kenntnisse auf diesem Gebiet geleistet. Donald Barron hat 1969 eben an der Universität von Florida den J.-Wayne-Reitz-Lehrstuhl für Biologie und Medizin der Fortpflanzung übernommen. Donald Barron gilt zusammen mit Sir Joseph Barcroft in Cambridge als der Vater des heutigen Kenntnisstandes in der Physiologie des Fötus.

Der Wissenschaft, der Forschung und dem Pferd gilt internationales Interesse, und im Bemühen darum werden Grenzen bedeutungslos. Eben dies bewirkt, daß die Forschungsarbeit in der Pferdeheilkunde solche Faszination ausübt und so reichen Lohn verspricht.

Im folgenden möchte ich auf die Beziehung zwischen Forschung und konkreten Verbesserungen in der Tiermedizin sowie in der Haltung und Zucht von Pferden eingehen. Die
Beispiele beziehen sich vornehmlich auf die perinatale Phase. Im Prinzip sind diese Ausführungen aber auch auf alle anderen Bereiche von Pferdezucht, Fohlenaufzucht und Pferdesport übertragbar.

\section{Anatomie der Forschung}

\section{Praktische Fragen und Antworten}

Tagtäglich sehen wir uns mit dem Problem konfrontiert, Krankheiten zu verhüten oder zu behandeln. Nehmen wir an, eine solche Erkrankung sei die Ursache für Sterilität, Abort oder Totgeburt bei einer Stute. Oder es geht um die Frage, warum ein Fohlen wenige Tage nach der Geburt plötzlich erkrankt, wo es doch bei der Geburt offensichtlich gesund war. Wir müssen bei der Suche nach Antwort immer nachforschen, wie verschiedene Hintergründe offensichtlicher Störungen miteinander verknüpft sein können. Eine Infektion des Genitaltraktes zum Beispiel kann bei der Stute die Konzeption verhindern, zum Abort oder zur Totgeburt führen, aber auch Grund für eine Erkrankung des Neugeborenen sein. Wir können ein Gesundheitsproblem nicht nur aus der Gesamtheit der sich gegenwärtig präsentierenden Symptome erklären, sondern wir müssen die gesamte Krankheitsgeschichte berücksichtigen. Welche Frage sich auch stellen mag, es ist die Forschung, von der die Antwort erwartet wird. Die Lösung eines Problems kann im Verständnis der Krankheitsursache, der Pathogenese oder der prädisponierenden Faktoren liegen oder auch in der Entwicklung von diagnostischen und therapeutischen Verfahren.

\section{Grundlagenforschung und praktische Forschung}

Wir hören oft von einer Trennung der Forschung in den Grundlagenbereich (akademische Forschung) und den Anwendungsbereich (klinisch-praktische Forschung). Grundlagenforschung läßt sich als Ansammlung von Wissen um seiner selbst willen beschreiben. Von praktischen Forschungsprogrammen dagegen erwarten wir anwendungsreife Ergebnisse in naher Zukunft.

Oft wird Grundlagenforschung zu praktischer Forschung, und die Unterscheidung ist nur eine Frage der zeitlichen Entwicklung. In den frühen 70er Jahren zum Beispiel hätte man Forschungsvorhaben zum Nachweis des Erregers der Contagious Equine Metritis (CEM) als Grundlagenforschung bezeichnet. Jedes Gremium, dem ein Antrag auf finanzielle Unterstützung eines Programms zur Untersuchung der mikroaerophilen Keime im Genitale der Stute und am Penis des Hengstes unterbreitet worden wäre, hätte es als solche betrachtet. Das Gutachten dieses Ausschusses hätte diese Arbeiten als zur Grundlagenforschung gehörig und ohne praktischen Bezug zu Problemen der Stutensterilität bezeichnet.

Als nun aber 1975 bei einer CEM-Epidemie der Nachweis eines mikroaerophilen Erregers gelang, waren die Untersuchungen an diesen Organismen plötzlich von höchst praktischem Wert und augenblicklich von größtem Interesse. Sie waren angewandte Forschung geworden.

Wir können also Grundlagenforschung und Forschung im klinischen Anwendungsbereich nicht sinnvoll trennen, da 


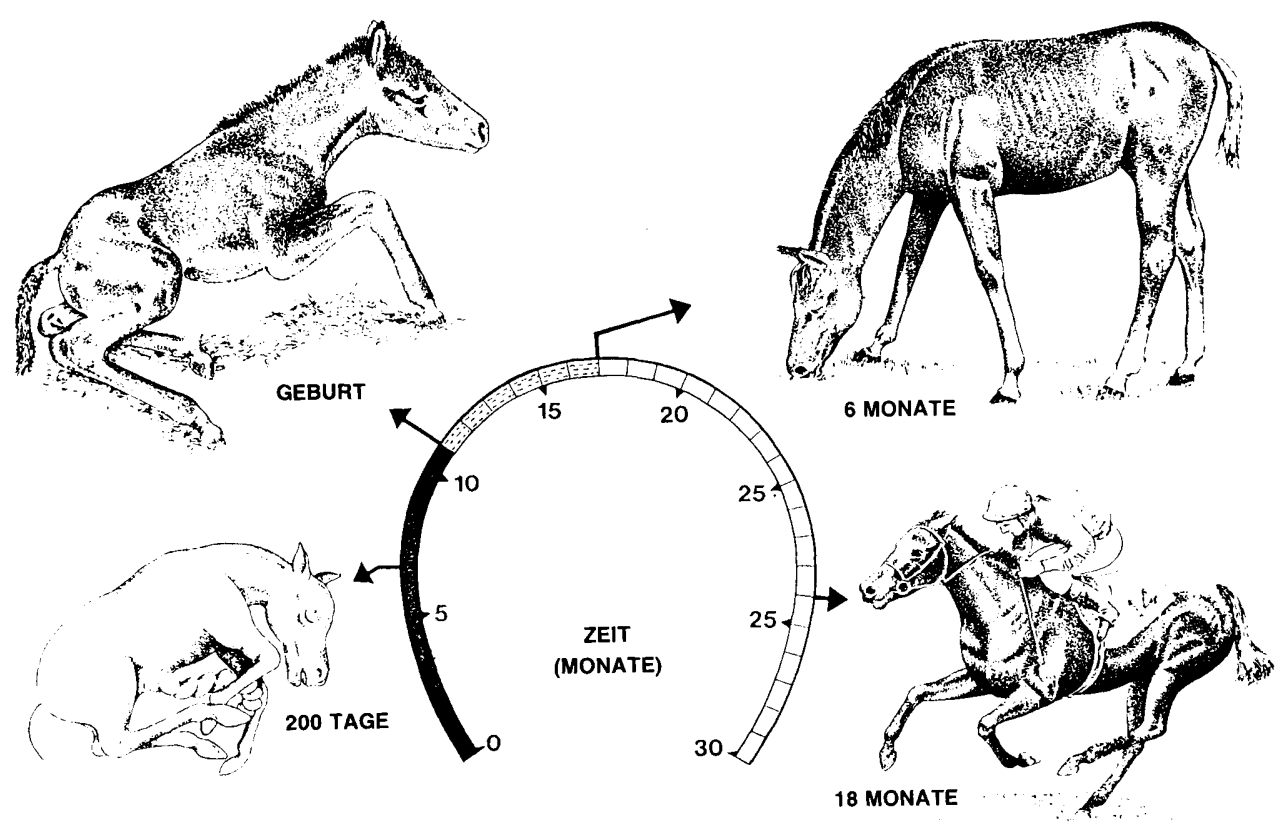

Abb. 1: Darstellung des kurzen Zeitraums zwischen Geburt und Einsatz als Rennpferd.

aus dem einen bald das andere werden kann. Einen wirklichen Unterschied gibt es nur zwischen guter und schlechter Forschung.

\section{Problemlösung}

Forschung dient der Lösung von Problemen, und Probleme bereiten Sorge, in unserem tierärztlichen Bereich z. B. dem Tierbesitzer, dem Trainer, dem Gestütsleiter, vielleicht auch demjenigen, der auf das Pferd gewettet hat oder, was in der Diskussion bedauerlicherweise oft in Vergessenheit gerät, dem Pferd selbst.

Was die Forschung betrifft, so sollten wir uns überlegen, wessen Interessen Vorrang haben. Die Tierbesitzer sind meist der Ansicht, sie selbst und ihre Interessen stünden im Vordergrund. Das ist verständlich, bietet aber nicht unbe- dingt eine gute Grundlage für die Auswahl der Fragen, denen die tierärztliche Forschung nachgehen soll. Die Tierbesitzer aber sind es, die für den Verlust der Zuchtfähigkeit oder der Konkurrenzfähigkeit bei Turnieren und Rennen finanzielle Verluste in Kauf nehmen müssen. Dies rechtfertigt, daß sie in der Reihe derer, die Lösungen für Probleme suchen, an erster Stelle stehen (Abb. 2).

Je nach Fragestellung, nach Art und Umfang des zu erforschenden Themenkomplexes sind die Kosten recht hoch. Im Vergleich zum Verlust jedoch, der entsteht, wenn für eine Krankheit keine Heilung möglich ist, sind die für die Forschung erforderlichen Mittel relativ gering. Der für veterinärmedizinische Untersuchungen über Herpesviren aufgewendete Geldbetrag z. B. bewegt sich im Bereich von mehreren hunderttausend Pfund Sterling, wohingegen das
Abb. 2.: Der Pferdebesitzer erwartet zu Recht, daß sich die tierärztliche Forschung in erster Linie mit der Lösung seiner Probleme befaßt, weil er sowohl die tierärztliche Liquidation als auch den finanziellen Verlust durch die Erkrankung an sich zu tragen hat. Letzterer ist oft um ein Vielfaches größer als die Beträge, die zur Erforschung des Problems nötig wären.

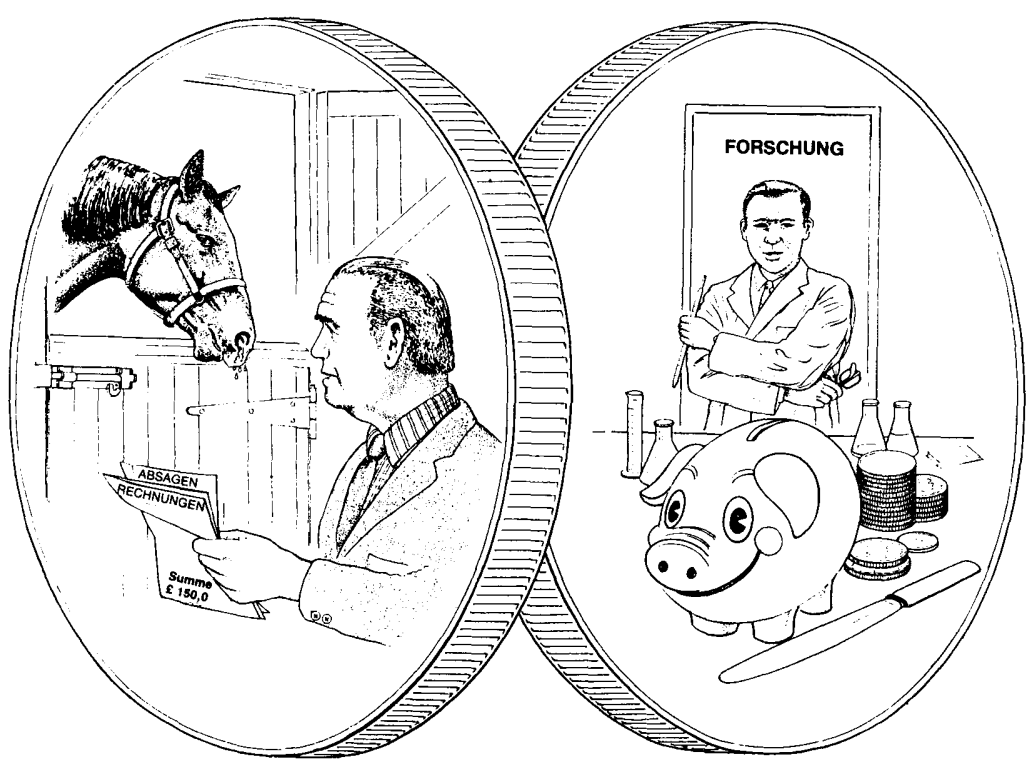


Abb. 3: Die Stufen von der Definition bis zur Lösung des Problems.

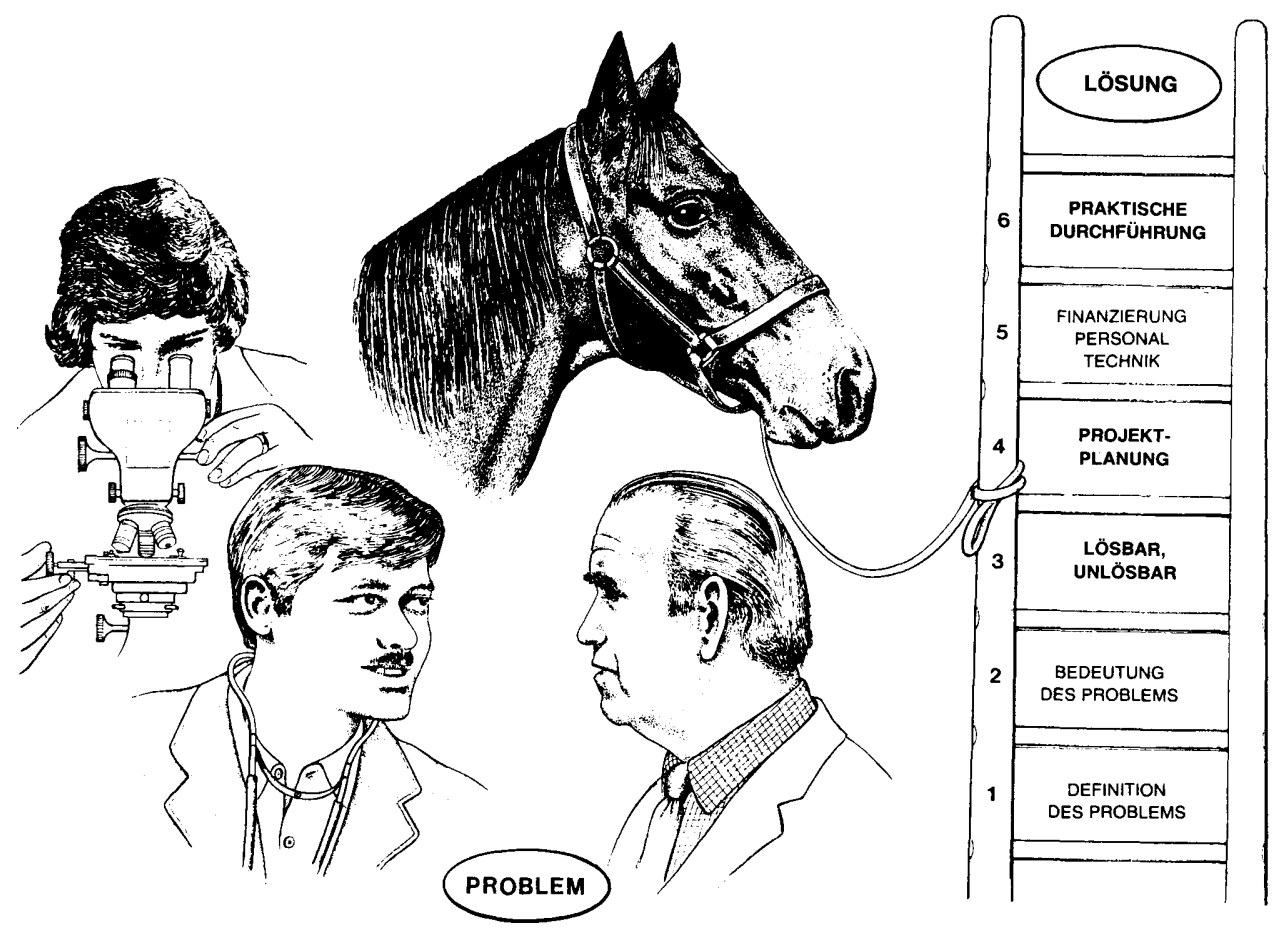

Herpesvirus den Tierbesitzern Verluste von vielen Millionen Pfund Sterling verursacht.

\section{Die 6 Stufen der Forschungsarbeit}

Zwischen der Erkennung eines Problems und dessen Lösung liegen, großzügig betrachtet, 6 Stufen (Abb. 3). Leider wird von seiten derer, die finanzielle Mittel für Forschungszwecke zur Verfügung stellen, oft übersehen, daß es Zeit in Anspruch nimmt, diese Stufen von der Definition einer Fragestellung bis zu deren Lösung hinter sich zu bringen. Forschungsvorhaben können sich im Einzelfall sehr in die Länge ziehen, insbesondere wenn die Entwicklung neuer Techniken erforderlich wird. Im günstigen Fall geht es so schnell wie bei der Eindämmung des CEM-Aus- bruchs in den 70er Jahren. Der Grund dafür, daß es nur eines kleinen Schrittes bedurfte, war die Möglichkeit der Anwendung humanmedizinischer Technologien zur Lösung eines veterinärmedizinischen Problems. Die Entwicklung der entsprechenden Verfahren war damals bereits abgeschlossen.

\section{Stufe 1: Definition des Problems}

Der erste Schritt in Richtung auf die Lösung eines Problems besteht in dessen Definition. Handelt es sich z. B. um eine einzelne Frage oder um einen Fragenkomplex? Dies zu entscheiden erfordert in der Regel eine exakte Diagnosestellung, nachdem man sich einen gründlichen Einblick in die Problematik verschafft hat.

Betrachten wir als Beispiel den Kreis respiratorischer Erkrankungen beim Pferd (Abb.4). Wir werden schnell zu
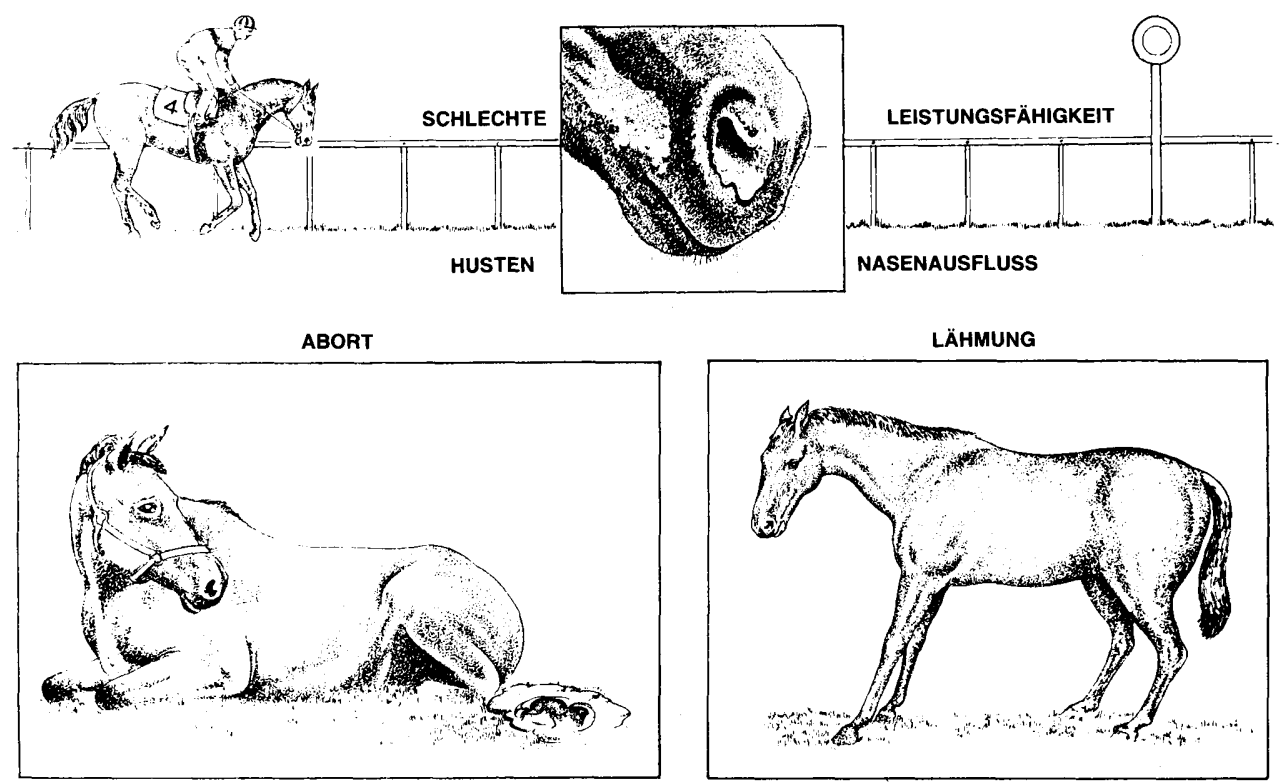

Abb. 4: Fragestellungen, deren sich die Forschung annehmen soll, bedürfen zuallererst der Definition des Problems. Nasenausfluß ist beispielsweise keine eigenständige $\mathrm{Er}$ krankung, sondern Symptom für die unterschiedlichsten Ursachen und Folgen. 
dem Schluß kommen, daß diese eine Vielzahl verschiedener Ursachen haben können. Es handelt sich in diesem Fall also nicht um ein einzelnes, sondern um eine Vielzahl von Problemen. Wenn wir nun mit unseren wissenschaftlichen Untersuchungen beginnen und so tun, als stünde hier ein Problem für sich allein, so sind wir mit unserer Arbeit bald an einem falschen Ende.

\section{Stufe 2: Bedeutung des Problems}

Der nächste Schritt besteht darin, die Bedeutung, d. h. die Häufigkeit und die Folgen des Problems festzulegen. Hier$\mathrm{zu}$ ist es von Bedeutung, sich einen Überblick über die tatsächlichen Verhältnisse zu verschaffen, unabhängig z. B. von der öffentlichen Meinung, die oft unzutreffend ist. Nur wenn wir sicher sein können, daß die praktische Bedeutung so groß ist, daß zur Klärung der Einsatz besonderer finanzieller Mittel gerechtfertigt ist, werden wir uns überzeugt an die Lösung des Problems machen.

\section{Stufe 3: Lösbar oder unlösbar?}

Als nächstes ist die Frage zu beantworten, ob das Problem mit den gegebenen Möglichkeiten zu lösen ist oder nicht. Es gibt Forschungsaufgaben, die auf dem jeweiligen Stand von Wissenschaft und Technik nicht zu klären sind. So hat es z. B. über 40 Jahre gedauert, bis wir der Früherkennung der Zwillingsträchtigkeit der Stute näherkamen. Diese Zeit verging seit den Anfängen der Entwicklung der Ultraschalltechnik sowie deren erfolgreicher praktischer Anwendung in der Humanmedizin bis zu ihrem heutigen Gebrauch in der Pferdemedizin. Die dabei entstandenen Verzögerungen beruhten teils auf technischen, teils auf wirtschaftlichen Schwierigkeiten.

Die Lösung eines Problems hängt oft davon $a b$, ob ein Schlüssel, möglicherweise ein Ergebnis der Grundlagenforschung gefunden wird. Unter Umständen ist die Beantwortung einer Frage ohne einen solchen bestimmten Schlüssel nicht möglich.

\section{Stufe 4: Projektplanung}

Die Projektplanung stellt ebenfalls einen wichtigen Schritt bei der Forschungsarbeit dar. Ist die Fragestellung klar, so müssen wir uns vergewissern, daß die Wege, die zur Antwort führen, genau festzulegen und die anzuwendenden Methoden ihrerseits gründlich erforscht sind.

\section{Stufe 5: Personelle, technische und finanzielle Versorgung} Hinsichtlich der personellen Besetzung eines Forschungsprogramms ist es oft unverzichtbar, Spezialisten aus anderen Disziplinen zu Rate zu ziehen. Möglicherweise handelt es sich dabei nicht um Tierärzte oder Personen, die sich mit Pferden befassen. Beim Versuch, z. B. neue Erkenntnisse über Rotavirusinfektionen beim Pferd zu gewinnen, ist es sinnvoll, sich des Wissens von Fachleuten zu bedienen, die auf diesem Gebiet bereits Erfahrung mit anderen Spezies sammeln konnten.

Bei der technischen Einrichtung ist es ähnlich. So erscheint es zweckmäßiger, bei der Erforschung der Physiologie der Milchdrüse Institute bei der Arbeit zu beteiligen, die sich in dieser Hinsicht mit Schafen und Rindern befassen, anstatt eine neue Einrichtung speziell für das Pferd zu schaffen. Selbstverständlich steht und fällt jede Forschungsarbeit mit den finanziellen Mitteln, die dafür zur Verfügung stehen. Darüber hinaus sollte eine Person die Untersuchungen koordinieren. Der Erfolg wissenschaftlicher Vorhaben hängt heute mehr und mehr von der Zusammenarbeit untereinander und mit anderen Disziplinen ab.

\section{Stufe 6: Praktische Anwendung}

Der letzte dieser Schritte vom Problem als solchem bis zu dessen Klärung muß die praktische Anwendung der Erkenntnisse sein. Dabei ist von größter Wichtigkeit die Antwort auf die Frage: Hilft das Ergebnis wirklich, das Problem zu lösen? Wenn dem so ist, gibt es irgendwelche Nebenwirkungen? Kommt es dann zur Erprobung der Ergebnisse am Tier, so stehen Kliniker, Praktiker und Pferdebesitzer in vorderster Front. Allzuoft arbeiten Wissenschaftler Methoden aus, die in der praktischen Anwendung nicht die erhofften Vorteile bringen.

\section{Methoden, Forschung zu betreiben} Grundsätzlich sehe ich vier Methoden, Forschung zu be-
treiben:

1. Empirische Forschung

2. Klinische Forschung

3. Experimentelle Forschung

4. Übertragung von Entwicklungen anderer Disziplinen auf die Pferdemedizin oder -haltung

\section{Empirische Forschung}

Darunter verstehe ich in diesem Zusammenhang die Durchführung von Beobachtungen am Tier in Praxis und Pferdehaltung und deren klinische Überprüfung. Praktische Tierärzte und auch mit der Haltung von Pferden befaßte Personen können hierbei mitarbeiten. Bei der Auswertung der Befunde bedarf es möglicherweise der Unterstützung durch Fachleute anderer Spezialgebiete. Solche Untersuchungen liefern uns Informationen über Häufigkeit und Verteilung von Krankheiten, deren Zusammenhang z. B. mit Haltung, Keimmilieu, Rasse oder Nutzung der erkrankten Tiere, Reaktionen auf Behandlungen, Prognose, Morbidität und Mortalität.

Wir können auf diese Weise Untersuchungen über die Sehnenkontraktur beim neugeborenen Fohlen anstellen, um Vorkommen, Einfluß von Erbfaktoren, Erkrankungen der Muttertiere während der Trächtigkeit und von deren Haltung und Fütterung zu ermitteln. Es würden dann Untersuchungen über Therapieerfolge und prophylaktische $\mathrm{Maßnahmen}$ folgen. Empirische Forschung bedeutet in diesem Zusammenhang im wesentlichen Beobachtung, Aufzeichnung, Sammlung und Vergleich von Veränderungen. Sie eignet sich zur Untersuchung von Krankheiten (z. B. der Septikämie des Neugeborenen), unphysiologischen $\mathrm{Zu}$ ständen (z. B. Sehnenkontraktur der Vordergliedmaßen) oder auch mehr allgemeinen Problemen wie wirtschaftliche Verluste (z. B. Dezimierung von Rennpferdebeständen durch Unfruchtbarkeit).

Mitglieder der tierärztlichen Fakultät von Gainesville ha- 
ben durch ihre Untersuchungen von Krankheitsfällen, beı denen eine spezifische Infektion des neugeborenen Fohlens vorlag, eine Vielzahl nützlicher Erkenntnisse gewonnen, wobei Anne Koterba und Barbara Brewer besondere Erwähnung verdienen. Dank ihrer Forschungen verfügen wir heute über eine bessere Diagnostik, können wirksamer therapieren und präzisere Aussagen über die Prognose bei der Septikämie des neugeborenen Fohlens geben. Sowohl die Morbiditäts- als auch die Mortalitätsrate wird so deutlich gesenkt.

Die Entwicklung eines Befundformulars, auf dem alle Veränderungen, die das erkrankte Fohlen aufweist, festgehalten und beurteilt werden, erlaubte den systematischen $\mathrm{Zu}$ gang zu einem Problem, bei dem man lange Zeit vor einer Fülle ungeordneter Einzelbefunde gestanden hatte. Auf diesem Sepsis-Formular wird zuallererst die Krankheitsgeschichte detailliert vermerkt, z. B. eine vorausgegangene Entzündung oder Infektion von Amnion oder Plazenta oder frühzeitiger Milchfluß der Stute.

Die klinischen Symptome sind natürlich wichtige diagnostische Indikatoren, aber sie können auch durch nichtinfektiöse Störungen wie das Maladaptationssyndrom des Neugeborenen beeinflußt sein. Zu den klinischen Erscheinungen zählen Schläfrigkeit, Lethargie, verminderte Saugkraft und die reduzierte Fähigkeit, die Saughaltung beizubehalten. Weitere Anzeichen sind Dehydratation, erhöhte oder subnormale Körpertemperaturen sowie injizierte Skleren. Spezifische Symptome einer Infektion wie Durchfall können hinzutreten. Die Veränderungen der Laborwerte bestehen vor allem in einer erhöhten oder verminderten Zahl neutrophiler Granulozyten, in einer Erhöhung des Fibrinogengehalts im Blut sowie einer reduzierten Immunglobulinkonzentration ( $\mathrm{IgG}$ ).

\section{Klinische Forschung}

Arzneimittelversuche, oft in Zusammenarbeit mit der pharmazeutischen Industrie oder anderen kommerziellen Herstellern, haben sich in der human- und veterinärmedizinischen Wissenschaft als klinische Forschung, die klare Ergebnisse verspricht, etabliert. Untersuchungen zur Methodik, zum Beispiel Entwicklung neuer chirurgischer Verfahren oder Tierhaltungsformen, nützen Tierarzt und Tierhalter in gleichem Maße.

Die klinische Forschung läßt den Vergleich einer Methode mit einer anderen $\mathrm{zu}$, wobei eine unbehandelte Kontrollgruppe Teil der Untersuchung sein muß. Dies erlaubt eine objektive Beurteilung unter Versuchsbedingungen. Diesen Untersuchungen sind immer ethische Grenzen gesetzt, da z. B. Fälle unbehandelt bleiben müssen, wo doch die erprobte Therapie möglicherweise hilfreich ist. Eine weitere Schwierigkeit besteht in der Voraussetzung konstanter Versuchsbedingungen. Korrekte und verwertbare Ergebnisse erfordern den weitgehenden Ausschluß von Störvariablen. Vergleichen wir die chirurgische Behandlung am Karpus des Pferdes mit einer unbehandelten Kontrollgruppe, so müssen wir sicherstellen, daß alle Pferde gleich gehalten und bewegt werden, daß sie gleich alt sind, daß der Erkrankungsgrad der gleiche ist usw.

Darüber hinaus ist für derartige Untersuchungen eine aus- reichende Zahl von Tieren erforderlich, um eine statistische Analyse rechtfertigen zu können.

Ich möchte als Beispiel eine Studie nennen, in der wir behandelte Tiere unbehandelten gegenübergestellt haben. Dabei arbeitete ich mit dem jungen deutschen Tierarzt Thomas Michel in der Zuchtsaison 1985 zusammen. Wir untersuchten die Wirkung von humanem Choriongonadotropin (HCG) zur Auslösung der Ovulation bei Stuten mit rektal festgestellt reifen Follikeln. Als Vergleich diente eine Gruppe mit Choriongonadotropin-Releasinghormon und eine weitere mit physiologischer Kochsalzlösung behandelter Stuten. Wir konnten zeigen, daß die Injektion von HCG eine bessere Wirkung hatte als die einer einzelnen Gabe von $\mathrm{GnRH}$ (wiederholte Gaben von $\mathrm{GnRH}$ sind offenbar wirksam). Die GnRH-behandelte Gruppe glich der Kontrollgruppe in dem Punkt, daß sich innerhalb von 48 Stunden nach der Injektion nur eine schwache, wenn überhaupt eine Wirkung in Form einer Ovulationsauslösung zeigte. Ein weiteres Ergebnis unserer Untersuchung war, daß unter den damaligen Haltungsbedingungen die Konzeptionsrate nach Verabreichung von HCG höher war als nach Applikation von GnRH oder bei der Kontrollgruppe. Allerdings ist dieser Effekt möglicherweise die Folge aus positivem Zusammenwirken von Haltung und tierärztlicher Betreuung und nicht so sehr die der HCG-Injektion allein.

\section{Experimentelle Forschung}

In experimentellen Studien werden Fakten über Krankheiten oder Wirkungen gesammelt: Fakten über die Wirkung von Medikamenten, Umwelteinflüssen usw. auf Krankheiten, Fakten über die Reaktion von Tieren unter kontrollierten Bedingungen, aus denen wir Informationen über Ursachen, Physiologie und Pathogenese einer Krankheit erhalten. Sind empirische und klinische Forschung auch ebenso bedeutsam und unverzichtbarer Teil der Forschung, so wird die experimentelle üblicherweise als die eigentliche Forschung betrachtet. Es werden zwei Arten unterschieden: in vitro, durchgeführt in mikrobiologischen, immunologischen, molekularbiologischen, virologischen usw. Labors, und in vivo, am lebenden Organismus. Experimentelle In-vivo-Forschung arbeitet mit Krankheitsmodellen oder mit physiologischen Bedingungen derselben Spezies. Beides läßt sich am Pferd durchführen, und ich werde es an 2 Beispielen erläutern: 1. Dauerkatheter in trächtigen Stuten und 2. das Modell der Frühgeburt beim Pferd. Mit experimentellen Untersuchungen lassen sich auch physiologische Vorgänge, z. B. der Kohlenhydratmetabolismus des Neugeborenen, studieren.

\section{Experimente an tragenden Stuten}

Ein wichtiger Bereich, in dem noch viele drängende Fragen offen sind, ist die Trächtigkeit und deren Zusammenhänge mit Physiologie und Pathologie des Fötus. Bei allen Versuchstierarten war ein wichtiger Schritt zu neuen Erkenntnissen auf diesem Gebiet das Anlegen von Verweilkathetern. Diese Technik wurde in den 30 er Jahren von Donald Barron entwickelt. Eines der wenigen, wenn nicht überhaupt das einzige Forscherteam, das sich mit dieser Technik befaßt hat, war das von Robert Comline, Marian Silver 
und deren Mitarbeitern von der Universität Cambridge. Sie haben die Technik von Barron übernommen.

Das Verfahren besteht darin, tragenden Stuten in Narkose Katheter in die Arteria und Vena umbilicalis des Fötus sowie in die Arteria und Vena uterina zu legen. Die Katheter verlaufen durch das Abdomen der Stute nach außen, wo sie in einer ledernen Tasche enden. Sie sind ständig mit gerinnungshemmender Flüssigkeit gefüllt, und bei Bedarf kann so Blut von Fötus und Uterus gewonnen werden.

Bis heute hat man diese Technik bei verschiedenen Tierarten einschließlich Schaf und Rind angewandt. Die Ergebnisse zeigen z. B., daß das fötale Fohlen eine höhere Blutsauerstoffkonzentration besitzt als das fötale Lamm. Wird bei der Stute die Sauerstoffkonzentration künstlich erhöht, so erreichen den Fötus größere Sauerstoffmengen, als dies beim Schaf der Fall ist, und der Sauerstoffspiegel steigt nicht wie beim Schaffötus nach Erreichen einer bestimmten Grenze nicht weiter an. Gleichgültig wieviel Sauerstoff dem Mutterschaf verabreicht wird, höhere Konzentrationen erreichen den Fötus nicht. Die Bedeutung dieses Unterschieds für das fötale Fohlen ist ungeklärt und bedarf weiterer Untersuchungen.

Eine weitere interessante Erscheinung, welche die Arbeiten von Marian Silver und ihren Kollegen gezeigt hat, ist, daß Futterentzug bei der tragenden Stute zum Ansteigen des Prostaglandinspiegels führt, des Hormons, das unter anderem für die Kontraktilität des Uterus und für die Blutversorgung von Uterus und Plazenta verantwortlich ist.

In Abhängigkeit von den Fütterungszeiten kommt es zu zirkadianen Schwankungen der Prostaglandinkonzentration im Blut. So ist der Spiegel vor der morgendlichen Fütterung der Stute höher als danach. Diese Schwankungen sind im späteren Trächtigkeitsabschnitt deutlicher als in der frühen Graviditätsphase.

Über den Einfluß der Fütterung unter normalen Haltungsbedingungen auf das Wohlbefinden des Fötus lassen sich bislang nur Vermutungen anstellen. Es ist zu hoffen, daß die Forschungsarbeit über die Physiologie der Trächtigkeit weitere Erkenntnisse darüber bringt, inwieweit die $\mathrm{Hal}$ tungsbedingungen die Gesundheit des Fötus beeinflussen und ihn schädigen bis hin zum Abort.

\section{Untersuchungen zum Woblbefinden \\ von Fötus und Neugeborenem}

Wir sollten uns stets vor Augen halten, daß das Wohlbefinden des Neugeborenen vom Wohlbefinden des Fötus abhängt, was wiederum eine gesunde Plazenta voraussetzt. Möglicherweise üben genetische oder sonstige innere Faktoren einen entscheidenden Einfluß auf dieses System aus. Sie sind durch die Chromosomenbindung zum Zeitpunkt der Befruchtung vorgegeben.

Es gibt jedoch auch äußere Faktoren, die sich ungünstig auf die Gesundheit und normale Entwicklung des Fötus auswirken. Hypoxie, Infektionen, Futtermangel oder Überfütterung, hormonelle und immunologische Dysfunktionen sind nur einige davon. Solche Einflüsse verursachen Störungen in der Plazenta und wirken direkt oder indirekt auf den Fötus. Die Folge sind verschiedenste Erkrankun- gen des Fohlens wie Septikämie, Maladaptationssyndrom, Skelettdeformierungen oder Hämolyse.

Die Gesundheit der Plazenta ist deshalb von überragender Bedeutung, weil eine Schädigung oder gestörte Funktion ihrerseits immer ein Ernährungsdefizit des Fötus zur Folge hat. Bei Neugeborenen zeigen sich dann Symptome wie Schwäche oder Untergewicht (Abb. 5).

Je nachdem zu welchem Zeitpunkt der Gravidität die Störung abläuft, wird die Entwicklung von Organen, Enzymsystemen usw. behindert. Das Fohlen leidet dann an dem, was als "distortion pathology" beschrieben wurde, das heißt mehr an der mangelhaften funktionellen Entwicklung als an der krankhaften Veränderung an sich.

Ist der Fötus z. B. während einer Phase, in der das Nerven-

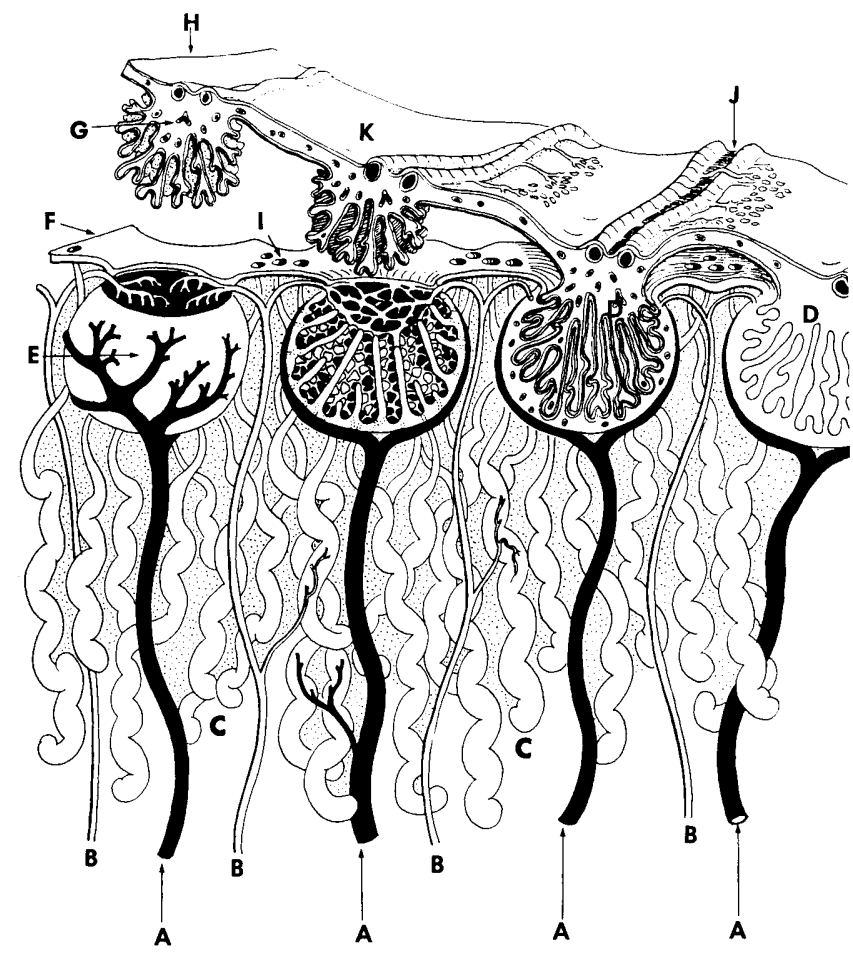

Abb. 5: Darstellung zweier Zustandsformen der normalerweise eine Einheit bildenden Plazenta und Uterus. Links zwei Mikrokotyledonen, herausgelöst aus dem Uterus. Comline und Silver entwickelten eine praktikable Technik, Blut aus Uterus und Plazenta gewinnen zu können.

A - Vene aus den Mikrokotyledonen (E und D); B - Arterie zu den Mikrokotyledonen; $\mathrm{C}$-Uterindrüsen mit Mündung des Ausführungsgangs bei I; $D$ und $E$ - Mikrokotyledonen; $F$ - Lumenseitige Uterusfläche; J-Plazentagefäße; G-Mikrokotyledonen der Plazenta.

system eine rasche Entwicklung durchläuft, ungünstigen Einflüssen ausgesetzt, so zeigt das Fohlen später möglicherweise Mangel an so lebensnotwendigen Strukturen wie Myelin, was sich in neurologischen Symptomen äußert. Diese Störungen im Sinne einer gehemmten funktionellen Entwicklung treten möglicherweise während der herkömmlichen pathologischen Untersuchung nicht zutage. Das Problem ist also mit der routinemäßigen Sektion nicht faßbar. Hierfür steht der Begriff „distortion pathology“. Einen Punkt, den wir noch streifen sollten, ist die enge Beziehung zwischen Plazenta und Entwicklung und Sekretion der Milchdrüse. Eine plazentare Störung erhöht das Risiko der verfrühten Euteranbildung und des zu frühen 
Milchflusses sowie des damit verbundenen Verlustes an Kolostrum. Diese Zusammenhänge bedürfen noch weiterer Untersuchungen.

Die als Frühgeburt und als Unreife bei normal langer Trächtigkeit bekannten Erscheinungen sind Folge von Störungen von Plazenta und Fötus. Die klinischen Anzeichen des Frühgeborenen sind zu kleine Körpergröße, Schwäche, die beim Aufstehen nach einem als normal anzusehenden Zeitraum von 2 Stunden nach der Geburt zum Ausdruck kommt, Hypoflexion der Fesselgelenke, seidige Haut und eine helle oder auch dunkelrote Zunge.

Der Ausdruck „geburtsunreif“ bezeichnet Fohlen, die nach physiologisch langer Gravidität Erscheinungen von Frühgeborenen zeigen. Diese Fohlen nennen wir auch depriviert, depriviert von normaler Plazentafunktion.

Frühgeborene und geburtsunreife Fohlen zeigen unter Umständen am zweiten Lebenstag normale Adaptationsreaktionen und werden kräftiger, oder aber sie entwickeln das, was man als „second day syndrome“ bezeichnet, zunehmende Verschlechterung des Allgemeinzustandes und Auftreten neurologischer und metabolisch-respiratorischer Störungen, was sich am zweiten Lebenstag entwickelt und mit dem Tod des Fohlens endet.

Ein experimentelles Forschungsprojekt, durch welches die Probleme von Frühgeburt und Geburtsunreife untersucht werden sollte, wurde in den späten 70er Jahren von einer Gruppe von Wissenschaftlern in Newmarket begonnen. Dazu gehörten Leo Jeffcott, Desmond Leadon und ich selbst. Später schlossen sich uns Jennifer Ousey und eine Reihe von Mitarbeitern anderer Institute, sowohl veterinär- als auch humanmedizinischer in Cambridge und anderswo, an. Das Unternehmen wurde mit einer Ponyherde durchgeführt, die im Animal Health Trust in Newmarket gehalten und finanziell vom Wellcome Trust, vom Horserace Betting Levy Board und vom Animal Health Trust getragen wurde.

Die Forschungsarbeiten umfaßten die Geburtseinleitung bei Stuten in verschiedenen Trächtigkeitsstadien und die Beurteilung des physiologischen bzw. pathologischen $\mathrm{Zu}-$ standes des jeweils geborenen Fohlens. Auf diese Weise standen uns Fohlen zur Verfügung, deren intrauterines Alter vor der künstlich eingeleiteten Geburt genau bekannt war. Wir konnten die Anzeichen von Frühgeburt beurteilen und mit den Fällen von Frühgeburt oder Geburtsunreife draußen in der Praxis vergleichen. Die wesentlichen Erkenntnisse aus diesen Untersuchungen waren folgende. Frühgeborene Fohlen zeigten: 1. Hypoflexion der Fesselgelenke; 2. niedrige Kortisolspiegel; 3. hohe ACTH-Spiegel; 4. keine Erhöhung des Kortisolspiegels nach Verabreichung von synthetischem ACTH; 5. entsprechend der Nebennierenrindenaktivität ein enges Neutrophilen-Lymphozyten-Verhältnis, was sich nach Verabreichung von ACTH nicht wie bei gesunden Fohlen ändert.

Die Ergebnisse unserer Untersuchungen zeigen, daß frühgeborene und geburtsunreife Fohlen an einer Nebenniereninsuffizienz leiden, d.h., die Nebennierenrinde reagiert nicht auf ihr natürliches Stimulans ACTH. Weiterhin können wir die Diagnose der Unreife auf das Verhältnis der Neutrophilen zu den Lymphozyten stützen und dessen Reaktion auf den ACTH-Stimulations-Test. Diesen Erkennt- nissen folgten Untersuchungen über die bestmögliche Therapie mit ACTH und Kortisol bei frühgeborenen und unreifen Fohlen.

Die Experimente galten auch der Untersuchung der Physiologie der Geburt sowie von Möglichkeiten, durch Untersuchung des Blutes auf Progestagene und Östrogene und des Eutersekrets auf Elektrolytgehalt Rückschlüsse auf die Geburtsreife des Fötus zu erhalten.

Das Projekt stellte eine kooperative und interdisziplinäre Zusammenarbeit einer Vielzahl veterinär- und humanmedizinischer Wissenschaftler dar. Es verdeutlicht, wie klinische Fragestellungen durch Experimente angegangen werden können. Wir befinden uns in der glücklichen Lage, im Rahmen unserer Forschungsarbeit am Pferd dieselbe Tierart als Versuchsmodell heranziehen zu können, an dem wir Fragen nachgehen können, denen wir draußen in der Praxis begegnen.

\section{4. Übertragung von Entwicklungen anderer Disziplinen auf die Pferdemedizin oder -baltung}

Die Technologie anderer Bereiche wie Physik, Chemie, Biochemie oder Pharmakologie ist möglicherweise in Chirurgie und innerer Medizin der Pferdeheilkunde anwendbar. Hier können Kosten, welche die eigene Entwicklung vergleichbarer Verfahren verursachen würde, eingespart werden. So sind viele Antibiotika in erster Linie für die Humanmedizin entwickelt worden, fanden aber später auch in der Pferdemedizin Verwendung. Molekularbiologische Methoden haben der Herstellung von Pferdeimpfstoffen gedient. Ein weiteres Beispiel ist die Ultraschalltechnik. Ihre Entwicklung wurde durch militärische Erfordernisse wie die der U-Boot-Fahndung angeregt. Nach der Fortentwicklung der Technik für die Bedürfnisse der Humanmedizin gelangt sie heute in der Pferdemedizin zur Untersuchung von Bindegewebsstrukturen z. B. bei Sehnenentzündungen oder beim Vollblutpferd zur Früherkennung der Zwillingsträchtigkeit zur Anwendung (Abb.6). Die Ultraschalldiagnostik ist ein Beispiel dafür, wie sich unter erheblichem Aufwand für fremde Disziplinen entwickelte Technik in die Pferdemedizin übernehmen läßt.

\section{Die Zukunft}

An dieser Stelle möchte ich 3 Aspekte unserer künftigen Forschungstätigkeit ansprechen. Es sind Bereiche, die dringend der weiteren wissenschaftlichen Untersuchung bedürfen zur Verbesserung der pferdemedizinischen Praxis insbesondere des neugeborenen Fohlens:

1. die Neurologie mit besonderer Berücksichtigung der Gefäßerkrankungen des Zentralnervensystems (Hämorrhagien), deren Behandlung und Verhütung;

2. die Physiologie und Pathophysiologie der Herz-Kreislauf- und Lungenfunktion, wobei die Entwicklung von Verfahren zur Behandlung schwerkranker Fohlen Vorrang hat;

3. die thermoregulatorischen Funktionen und die Verdauung sowie deren Zusammenhang mit den verschiedenen Haltungsformen. Wir müssen auch unsere Kenntnisse in bezug auf die Darmfunktion erweitern, um Fort- 
schritte in der Therapie und Intensivüberwachung kranker Fohlen zu erzielen.

\section{Neurologie}

Die Pathogenese subarachnoidaler und intrazerebraler Blutungen und ischämischer Nekrosen im Gehirn ist bei der Suche nach Wegen zu Verhütung und Heilung von besonderem Interesse und vorrangiger Bedeutung.

$\mathrm{Zu}$ den neurologischen Symptomen zählen Krämpfe, gesteigerter Extensorentonus, Koma, Zwangsbewegungen und zielloses Umherirren. Die Blutungen als Ursache dieser neurologischen Symptome bzw. Verhaltensstörungen
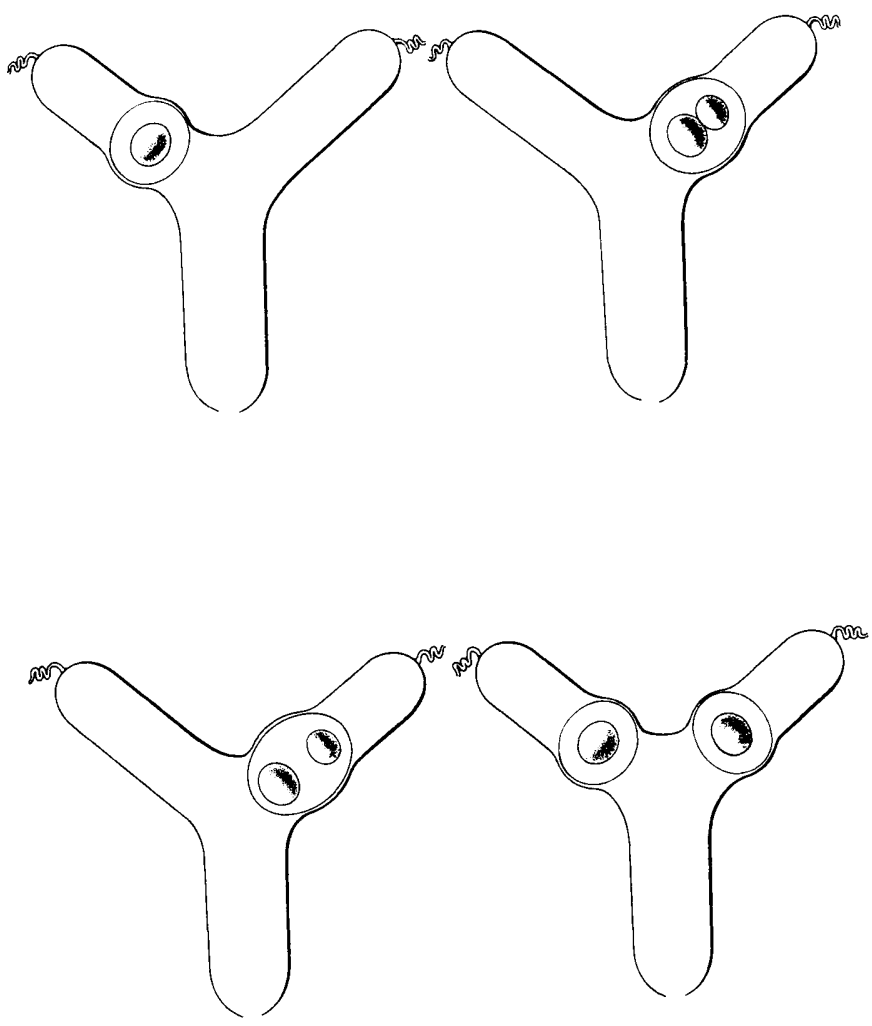

Abb. 6: Mit Hilfe der Ultraschalltechnik können wir die Lage des Embryos im Uterus sichtbar machen und schon 2 Wochen nach dem Deckakt klären, ob eine Zwillingsträchtigkeit vorliegt oder nicht.

entstehen im Großhirn, Kleinhirn oder Rückenmark. Auch gestörte Kreislauffunktionen können sich auf das Nervensystem auswirken. Während der Geburt kann sich das Fohlen Rippenfrakturen oder eine Herzquetschung zuziehen, oder es erleidet Sauerstoffmangel. Die Ereignisse können allein oder kombiniert zur Entstehung von Hirnblutungen beitragen.

Exakte Diagnostik und kritische Untersuchung des Patienten sind unabdingbare Voraussetzung für Fortschritte bei der Lösung des Problems. Adams und Mayberw von der Universität von Florida haben sehr zur Weiterentwicklung beigetragen, indem sie ein Untersuchungsprotokoll entwarfen, an Hand dessen sich eine exakte neurologische Diagnose stellen läßt. Ihre Arbeit ist ein Beispiel für die sogenannte empirische Forschung. Um Antwort auf einige interessierende Fragen nach Ursache und Entstehung von neurologischen Schädigungen zu finden, werden aber auch experimentelle Versuchsprogramme erforderlich sein.
Herz- und Lungenfunktion

Die Herz-Kreislauf- und Lungenfunktion bilden die Lebensgrundlage eines jeden von uns. In der Neugeborenenphase erfordert der Übertritt vom fötalen ins unabhängige Leben grundlegende kardiovaskuläre und pulmonale Anpassungsvorgänge. Es verwundert nicht, daß viele Neugeborene hierbei Unterstützung benötigen. Wissenschaftler wie Kosch, Koterba und Webb von der Universität von Florida oder Rose von der Universität von Sydney haben auf diesem Bereich große Fortschritte erzielt.

Jede unterstützende Maßnahme beruht auf den grundlegenden Prinzipien von Ventilation, Perfusion und Diffusion, d. h., sauerstoffhaltige Luft strömt in die Lunge (Ventilation), der Sauerstoff tritt in den Blutkreislauf über (Diffusion) und wird zu den Geweben transportiert, wo er im Prozeß der Energiegewinnung und Aufrechterhaltung lebenswichtiger Vorgänge verbraucht wird. Kohlendioxid wird in entgegengesetzter Richtung zur Lunge transportiert, wo es aus dem Blut in die Alveolen diffundiert und ausgeatmet wird. Beim neugeborenen Fohlen besteht möglicherweise eine Störung in Form einer Shunt-Bildung. In diesem Fall tritt Blut von der rechten auf die linke Herzseite und erreicht den arteriellen Kreislauf, ohne Sauerstoff aufgenommen zu haben. Die Shunt-Wirkung entsteht entweder in nicht ventilierten Lungenbezirken (physiologischer Shunt) oder als anatomischer Shunt über fötale Öffnungen im Bereich des Herzens, namentlich über Ductus arteriosus und Foramen ovale (Abb.7). Eine weitere Schwierigkeit für das Neugeborene besteht darin, daß im Sinne eines reibungslosen Geburtsverlaufs sein Brustkorb stärker nachgeben muß als der des Erwachsenen. Während der Fötus durch den Geburtskanal tritt, hat sich der Thorax diesem in seiner Form anzupassen. Man dachte bisher, der Brustkorb des Fohlens gebe leichter nach, sei weniger steif als der des Erwachsenen. Koterba und Kosch haben jedoch nachweisen können, daß dies nicht der Fall ist. Jedenfalls bedarf es weiterer Forschungsarbeit, um herauszufinden, ob die Atmungsmechanik des kranken neugeborenen Fohlens deshalb gestört ist, weil es frühgeboren oder ge-

\section{LUNGE}

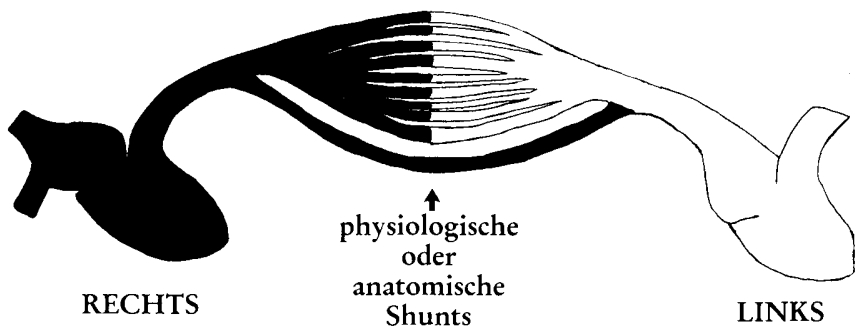

Abb. 7: Beim physiologischen Shunt fließt Blut durch nicht ventilierte Lungenbezirke, beim anatomischen Shunt durch Öffnungen im Bereich des Herzens (Ductus arteriosus, Foramen ovale).

burtsunreif ist, weil es infolge seiner Krankheit länger liegt oder etwa weil es durch Krämpfe oder allgemeine schlechte Verfassung zu schwach zum Atmen wird.

Die Unterstützung der Atmung hat sich in der Praxis als unverzichtbar bei der Intensivüberwachung erwiesen. Die Entwicklung des Nasotracheotubus durch Webb stellt einen einfachen, aber bedeutsamen Fortschritt in der Thera- 
pie respiratorischer Insuffizienz des neugeborenen Fohlens dar. Weiterhin ist eine Verbesserung dadurch zu erzielen, daß das Fohlen nicht in Seiten-, sondern in Brustlage gehalten wird. Diese Erkenntnis ist wiederum ein Beispiel für fruchtbare empirische Forschung. Durch systematische Beobachtung in der Praxis, Führung und Auswertung von Untersuchungsprotokollen, in diesem Fall durch Rose in Sydney und Kosch in Florida, ist es gelungen, einen wichtigen Fortschritt zu erzielen. Bei der Weiterentwicklung von Maßnahmen zur Unterstützung der Atmungsfunktion werden auch Einrichtungen zur kontinuierlichen Sauerstoffüberwachung beim Fohlen erforderlich. Zum heutigen Zeitpunkt ist eine einfache, zuverlässige und wenig invasive Methode hierfür nicht bekannt.

\section{Thermoregulation - Nabrungsaufnabme - Darmfunktion}

Auf den Gebieten der Thermoregulation, Ernährung und endokrinen Reaktionen sind weitere wissenschaftliche Studien dringend erforderlich, um die Behandlung unserer Krankheitsfälle zu verbessern. Das Leben im Uterus, in dem das Muttertier für Wärme und Ernährung sorgt, steht in scharfem Kontrast zur Situation des Neugeborenen. Das Fohlen muß die Nahrung selbst zu sich nehmen, und der Magen-Darm-Trakt übt erstmals seine Verdauungsfunktionen aus. Es muß durch ausreichende Energieproduktion seine Körpertemperatur konstant hoch halten. Eine Möglichkeit, diesen Fragenkreis wissenschaftlich anzugehen, besteht in der Messung des Sauerstoffverbrauchs bei verschiedenen Umgebungstemperaturen und bei unterschiedlich aufgenommenen Nahrungsmengen. Dies ist ein junges Forschungsprojekt, von dem man in naher Zukunft hören wird. Die erwarteten Ergebnisse werden, wie wir hoffen, die Versorgung des Neugeborenen hinsichtlich der Thermoregulation und Ernährung verbessern und Probleme wie Blähungen und Darmlähmungen verhindern helfen. Letzteres sorgt häufig genug für Enttäuschung und Ernüchterung beim Versuch, das kranke Fohlen zu ernähren.

\section{Schlußfolgerungen}

Die Verbesserung der tierärztlichen Versorgung von Stute und Fohlen sowie der Pferdehaltung und Gestütsführung war das Ziel von Jobn und Sandy Hartigan. Es ist unser aller Ziel. Die Rolle, welche die Forschung hierbei spielt, habe ich in diesem Artikel zu beschreiben versucht. Diejenigen von uns, die an Forschungsvorhaben arbeiten, sollten sich denen gegenüber verpflichtet fühlen, die die finanziellen Mittel dafür bereitstellen, und gegenüber denjenigen, in deren Auftrag sie arbeiten. Wir haben jederzeit sicherzustellen, daß die Themen unserer wissenschaftlichen Untersuchungen sinnvollen Bezug zu den Problemen der Praxis haben. Ist dies der Fall, so wird das Ergebnis immer nützlich sein. Die finanziellen Mittel, die Zahl der Forschenden und die technischen Möglichkeiten sind, was die Forschung am und für das Pferd angeht, zu begrenzt, als daß wir es uns leisten könnten, einerseits Chancen ungenutzt zu lassen und andererseits Zeit und Mittel zu verschwenden.

Ich denke, ein guter Schluß sind die Worte von John Masefield: „Adventure on! For from the littlest clue arose whatever worth Man ever know.“

Peter Rossdale, PhD, MA, FRCVS

Beaufort Cottage Stables

High Street, Newmarket

Suffolk, England

\section{Kurzreferat}

\section{Der Einsatz von Ultraschall zur Erkennung einer Arteria-Iliaca-Thrombose bei Pferden}

(Use of Ultrasonography for the Dedection of Aortic-Iliac Thrombosis in Horses)

Virginia W. Reef, Kate A. W. Roby, W. Richardson, Wendy E. Vaala und Janet K. Johnsten (1987)

JAVMA $190,286-288$

Im Rahmen dieser Arbeit wurde an 18 Pferden eine diagnostische zweidimensionale Ultraschalluntersuchung der A. iliaca und dem caudalen Aortenabschnitt durchgeführt. Alle 18 Pferde zeigten eine Lahmheit der Hinterhand, die nach Belastung einsetzte.

Der Ultraschalluntersuchung ging immer eine vollständige Lahmheitsuntersuchung einschließlich rektaler Befunderhebung voraus.

Dabei ergab sich, daß in 5 von 18 Pferden ein Thrombus oder Plaque diagnostiziert werden konnte, wobei bei 2 der 5 Pferde der rektale Befund unauffällig war und der Thrombus nur mit Hilfe der diagnostischen Ultraschalluntersuchung entdeckt werden konnte. Die Autoren kommen zu dem Schluß, daß der Einsatz von Ultraschallgeräten eine schnellere und leichtere Diagnose, besonders in der Entdeckung von Aneurismen, Aortenthromben und bei Plaques in der Arteria carotis, bietet und so eine schnelle und erfolgversprechende Therapie eingeleitet werden kann. 